\title{
Turbulent Flowfield Analysis in a Bluff-Body Burner Using PIV
}

\author{
Nattan Roberto Caetano, Flávio Tadeu van der Laan \\ Department of Mechanical Engineering, University Federal of Rio Grande do Sul, Porto Alegre, Brazil \\ Email: nattan@ufrgs.br
}

Received November 29, 2012; revised January 15, 2013; accepted January 23, 2013

Copyright (C) 2013 Nattan Roberto Caetano, Flávio Tadeu van der Laan. This is an open access article distributed under the Creative Commons Attribution License, which permits unrestricted use, distribution, and reproduction in any medium, provided the original work is properly cited.

\begin{abstract}
The structure of inert turbulent flows, stabilized in a Bluff-Body burner, is studied considering different volumetric flows for Nitrogen jet and annular air in coflow configuration. Flowfield analysis on Bluff-Body burner is essential to improve the knowledge about this burner, which plays an important role in industrial applications. Thus, vector velocity field is performed, employing Particle Image Velocimetry technique. Also, an uncertainty analysis is performed considering parameters involved in this technique yielding $6 \%$ to velocity measurements. The acquired information produces the results based in flowfield structure, which are presented in terms of statistical momentum and Reynolds stress, in which Boussinesq Hypothesis is considered to incompressible flows. However, this hypothesis fails in certain conditions. In this way, is possible to comprehend and provide experimental data from the turbulent effects on the flowfield and also contribute to predict the combustion flows, in order to enable the validation and develop numerical models.
\end{abstract}

Keywords: Particle Image Velocimetry; Turbulent Flowfield; Experimental Uncertainty; Boussinesq Hypothesis

\section{Introduction}

The aim of the work is study, experimentally, turbulent flowfields stabilized on the Bluff-Body burner. Therefore, measurements are performed using non-intrusive optical technique in order to yield the velocity field, employing PIV. This work intends to produce results which will allow apply advanced post-processing methods. So that, main experimental results are presented in order to verify the flowfield structures characteristics, based in Boussinesq Hypothesis. The knowledge yielded constitutes a data base which enable developing and validation of numerical models.

The present work emphasis is given by using the technique which allows measure the turbulent flowfield properties. Moreover, relevant aspects are approached, data and statistical results processing. Velocity measurements are necessary to investigate the turbulent flowfield structure and flow stabilization. However, this work focus on the analysis of the flowfield dynamic influence on the turbulence using velocity measures via Particle Image Velocimetry PIV [1,2]. Measurements of vector velocity field allow to perform the flowfield characterization by the analysis of the results from statistical momentum, as well as, through the flowfield Reynolds stresses tensor.
The flowfield stability depends on the interactions between dynamic, mixture and, chemical of the fluids considered. In this way, the velocity field structure plays an important role on the numerical models development. However, is a determinant factor in works which intend to characterize the different flowfield structures. Thus, both the diagnostic method purposed, followed by an image post-processing, enriched the range of statistical information available, such as a contribution toward the information database creation, which allows comparisons and numerical models calibration [3].

About parameters of the measure technique applied in turbulent flowfield based on lasers, a trend is observed. The capture rate used to measure reaching about $\mathrm{kHz}$, in one plane [4]. These measures covered small interrogations areas, about some $\mathrm{mm}^{2}$, due the lasers energy limitations. The size of the measurement window is smaller than the normal techniques are able to capture, circa 100 $\mathrm{cm}^{2}$. Nowadays, the works perform 3D vector field velocity, which is measured simultaneously with one scalar, more often, $\mathrm{OH}$ or $\mathrm{CH}$, or temperature [5,6]. High speed equipment is employed in order to investigate turbulent effects using information taken in high temporal resolution, in one or more planes [7]. Therefore, the results are 
described in terms of two first statistical momentums from the aero-thermochemical measures, as well as, advanced post-processing concerning the probability density function evolution and its derivatives, as vorticity or strain and stress rates [8].

\section{Experimental Approach}

\subsection{Burner Configuration}

The Bluff-Body burner used in this work was designed for the purpose of cover a broad operation range [9]. This type of burner is relevant in several engineer applications, including industrial flowfield problems, because is able to stabilize flames in different combustion regimes on its wake zone. This burner allows details studies about interaction between turbulence and chemical kinetic by using optical measuring techniques due the broad access, moreover, the high symmetry and simple boundary conditions became easy the numeric solutions algorithm. The burner dimensions was choose in order to obtain a large difference between thickness of laser light plane and jet diameter, 0.5 and $7.1 \mathrm{~mm}$, respectively. The fuel supply channel have $150 \mathrm{~cm}$, ensuring the fully flowfield development. The wind tunnel is $200 \mathrm{~mm}$ from which the environment air flowfield exits surrounding the central bluff-body with $60 \mathrm{~mm}$ diameter. The duct length is $1 \mathrm{~m}$, up a turbulence generator grid, which has $12 \mathrm{~mm}$ of diameter holes, separated $10 \mathrm{~mm}$ each other to generate turbulence, used in order to homogenize the flowfield. The interrogation window size is $120 \times 90 \mathrm{~mm}^{2}$ upward to the burner surface, which is centered in the fuel jet. Thus, this burner produces a turbulent flowfield containing a concentric central jet to an annular air exit around of the central body. To provide the air a fun (Deltra, VC400 ) is used, which yield up to $10 \mathrm{~m} / \mathrm{s}$ at the maximum frequency rotation, $60 \mathrm{~Hz}$. The top boundary of the flowmeter (OMEL, 3P5-0401V01) is $3 \mathrm{Nm}^{3} / \mathrm{h}$. Thus, the maximum fuel velocity is about $30 \mathrm{~m} / \mathrm{s}$ in standard temperature and pressure conditions.

\subsection{PIV Technique}

A double pulse Nd:YAG laser (Quantel, Twins) is used as source to illuminate the particles seeded in the flowfield by a laser light sheet of $532 \mathrm{~nm}$ and $0.5 \mathrm{~mm}$ of thickness. The cavities of laser are independent, allowing input a time interval $(d t)$ between two shoots. A camera (LaVision, Image Intense) captures the Mie scattering of laser light from the particles on a $1376 \times 1040$ pixel matrix, of 12 bit, with $6.45 \mu \mathrm{m}$ pixel size and $70 \%$ of quantum efficiency close of $532 \mathrm{~nm}$ wavelength, yielding 4 kbits of gray level contrast. Scattered photons are transmitted by a band-pass filter, centered in $532 \pm 5 \mathrm{~nm}$, which are focused on the CCD sensor by a lenses ensemble (Nikon, Nikkop, f/1.4, $50 \mathrm{~mm}$ ).
Tracer particles seeded in flowfield in order to apply PIV should attend three fundamental requirements. First, particles should have minimum dimensions to be able to scatter the incident laser light. Second, particles should follow the flowfield turbulent buoyancies. Third, particles should have melting point above the flowfield temperature [10]. The accuracy in the determination of the tracer particles dimensions plays an important role in the measurements uncertainty calculus in PIV [11]. PIV applications in gas phase flowfields require smaller particles and light power enough in order to the scattering intensity sensitizing the CCD camera, so that, the signal/ noise relation is sufficient to produces quality images. These requirements are crucial to the PIV employment high interference cases. The compromise between particle size and light scattered intensity was valued, leading the choice of Titanium Dioxide $\left(\mathrm{T}_{\mathrm{i}} \mathrm{O}_{2}\right)$ particles, with 1 $\mu \mathrm{m}$ diameter, which are often used and recommended for this purpose by the literature $[2,11,12]$.

The density of particles should be selected toward ensure the velocity measures within the expected range. Thus, both, the dimension and the density of the tracer particles have direct influence on the measurements uncertainty. In particular, the relaxation time of the particles when submitted to the flowfield velocity buoyancies should be smaller than the characteristic buoyancy time. Tracer particles dimension choice is the main parameter to the particles follow correctly the flowfield buoyancies. The relaxation time, $t_{s}$, is a convenient amount toward estimate the trend that particles have to keep in equilibrium within the flowfield. The ideal particle size can be estimated from the relaxation time of a particle in a fluid, which is obtained from the Stokes equation [1],

$$
d_{p}=\frac{18 \mu t_{s}}{\rho_{p}},
$$

where $d_{p}=1 \mu \mathrm{m}$ is the mean particles diameter, $\mu=17$ $\times 10^{-6} \mathrm{~Pa} . s$ is the kinematic viscosity of the air and $\rho_{p}=4.230 \mathrm{~kg} / \mathrm{m}^{3}$ is the specific mass of the $\mathrm{TiO}_{2}$, the particle material. However, this time is about $14 \mu \mathrm{s}$, i.e., the particles are able to follow buoyancies in the flowfield of about $70 \mathrm{kHz}$, regarding the conditions used in this work.

The velocity vector field is calculated by processing the pairs of particle images. In this work 1000 pairs of 4 $\mathrm{Mb}$ images was taken, in $3 \mathrm{~Hz}$ of acquisition rate. This cadence is not enough to describe the turbulent fluctuations of the flowfield, but is a limitation of the PIV system hardware. The processing is done by a dual core PC takes about 7 hours in multi-pass mode, two passes; decreasing window, $64 \times 64$ to $32 \times 32$ pixels; and overlap of $50 \%$ and $75 \%$, respectively to each pass, also applying the Whittaker reconstruction method $[13,14]$. These conditions were chose because yield better correlations and 
spatial resolutions, producing 5590 vectors spaced out $1.5 \mathrm{~mm}$.

\subsection{Calibration of the Technique}

The commercial software LaVision DaVis recognizes the pattern of points in a special plate designed as a calibration target, using an algorithm of search and recognizing. After that, a grid of known dimensions appears over the image in order to attribute dimensions to the pixels. The configuration set for this work the mean pixel diameter is $90 \mu \mathrm{m}$, with $0.5 \%$ of uncertainty.

A TSI, APS 3320 Particle Sizer equipment was used to measure the particles dimension and concentration in the flowfield. The suitable amount of particles to the experiment was determined by the direct visualization of the particle distribution in the Mie scattering images. The results to both, jet and annular flowfields, are $1 \mu \mathrm{m}$ of particles diameter with a low dispersion $(\sigma=0.3)$.

A Dwyer, S471 DTA hotwire anemometer, 2.5\% of uncertainty, calibrated by Skilltech, was used to measure the velocity of the annulus air in the burner flowfield. The results were compared with the PIV results in order to verify the accuracy level. The measurements were taken in two positions up wise of the burner surface, at the edge of the bluff-body and $100 \mathrm{~mm}$ higher, yielding 8.0 and $7.8 \mathrm{~m} / \mathrm{s}$, respectively. The averages of the velocities measured by PIV in these positions are exactly the same, within the equipment uncertainty.

\subsection{Measurement Uncertainty}

The uncertainty in PIV measurements involves topics which affect the accuracy, as the sources related and briefly discussed in sequence, 1) refraction index gradients, 2) non-homogeneity in particle distributions, 3) particle image size and, 4) information processing. The maximum differences in the refractions index by the air and Nitrogen is about $1 \%$, as the thickness of the laser light sheet, about $500 \mu \mathrm{m}$, is 5 times higher than the pixel size, the maximum distortion yielded by the refraction index gradients, $u_{\nabla n}$, is $2 \%$.

The non-homogeneity in particle distributions, due the difference between gases density and viscosity, produces fails in the correlation maps, causing errors in the processing and, consequently, yielding spurious vectors. The uncertainty associated to the particle dispersion fails, $u_{f}$, is higher in the wake region, as expected, because is the recirculation zone. The particle concentration in the wake region is $3 \%$, maximum, lower than the jet and annular regions yielding an uncertainty of the same order of magnitude.

The contribution of the particle image size can influence to the uncertainty in PIV measurements in two aspects. First, regarding the fidelity in follow the flowfield buoyancies. Second, is about the images processing, which correlation depends on the particle image dimensions in the frames and of the respective displacements. A study about the particle displacements was performed applying numerical simulations [1], in order to evaluate the influence. Regarding the configuration used to perform the present work, comparing with the results achieved by the simulations in the literature, the ideal size of the particle image is 1.5 pixel. The beam steering is also an important factor in the signal to noise ratio. The window size has similar importance because the uncertainty increases to smaller windows. The particle image diameter, $d_{i}$, is determined by,

$$
d_{i}=\sqrt{\left(M d_{p}\right)^{2}+d_{d i f f}^{2}},
$$

where $d_{\text {diff }}=2.44 f_{\#}(M+1) \lambda$ is the minimum diameter of the light diffracted by particles, $f_{\#}=11$ is the ratio between focal length and aperture diameter of the lenses in a camera, $\lambda=532 \mathrm{~nm}$ is the laser light wavelength and $M=0.10$ is the image magnification. The effective diameter, $1 \mu \mathrm{m}$, of the particles used in this work was measured using TSI Particle Sizer equipment. Therefore, the mean particle image diameter has 4 pixels, yielding an uncertainty of about 0.1 pixel, i.e., $u_{d_{i}}= \pm 3 \%$ to processing windows of $32 \times 32$ pixels.

The uncertainty brought by the information processing depends on the several sources, the correlation between image objects in the frames and the subpixel adjustment. The dispersion of the results around the maximum points in the correlation maps, calculated by the software, LaVision $\mathrm{DaVis}$, is 3 pixels in the wake regions, where the correlation is weak. Thus, is possible to estimate a maximum uncertainty due the correlation process as $u_{\text {corr }}=$ $5 \%$. The value of the error due the subpixel adjustment achieved by simulations reaches $u_{s p}=3 \%$ [1].

These sources of uncertainty in the PIV technique was detailed analyzed [2], whereas the maximum total uncertainty, i.e. in the recirculation zone, is calculated using the Kline-McClintock method $[15,16]$, considering the contribution of each source discussed before. These results were propagated toward achieve the total measurement uncertainty, as shown in the following Equations (3) and (4).

$$
\begin{aligned}
u_{P I V} & = \pm\left[\left(u_{\nabla n}\right)^{2}+\left(u_{f}\right)^{2}+\left(u_{d_{i}}\right)^{2}+\left(u_{c o r r}\right)^{2}+\left(u_{s p}\right)^{2}\right]^{1 / 2} \\
u_{P I V} & = \pm\left[(2 \%)^{2}+(3 \%)^{2}+(3 \%)^{2}+(5 \%)^{2}+(3 \%)^{2}\right]^{1 / 2} \\
& =6 \pm \%
\end{aligned}
$$

\section{Results}

In this section are presented: 1) the experimental results 
obtained by this work, 2) the analysis of the velocity results yielded by PIV in order to characterize the flowfield dynamic structure produced in a Bluff-body burner. Thus, the jet Nitrogen and annular air flow conditions are related in the Table 1 for the two cases involved in this work. The mean jet velocity value is also introduced, in order to improve the analysis. Also, the time intervals between frames in PIV are presented for each case.

Each studied case presented in this section involves three instantaneous overlapped by the respective vector velocity field, obtained on the burner symmetry plane. The behavior of the mean flowfield shown by stream lines is analyzed in the sequence, both obtained from 1000 instantaneous images. Moreover, are discussed the behavior of the mean velocity components, transversal and longitudinal, and the Reynolds tensor based in the Boussinesq hypothesis principle. These quantitative results are presented in form of graphics where the values was extract from 7 transversal positions in $10 \mathrm{~mm}$ of interval between each other upward of the burner surface.

\subsection{Boussinesq Hypothesis Analysis}

Turbulence modeling, generally, employs the Boussinesq hypothesis in order to determine the Reynolds tensor, through the relation with the mean strain rate in the flowfield as follow [17],

$$
-\overline{V_{i}^{\prime} V_{j}^{\prime}}=2 v_{t} S_{i j}-\frac{2}{3} K \delta_{i j},
$$

where $v_{t}$ is the turbulent viscosity, $K=1 / 2\left(\overline{V_{i}^{\prime} V_{j}^{\prime}}\right)$ is the turbulent kinetic energy, $\delta_{i j}$ is the Kronecker's delta and $S_{i j}$ is the mean strain rate tensor. This tensor can be simplified to the flowfields which has revolution symmetry, as the flowfield produced by the burner considered, which implies that, if Boussinesq hypothesis is valid, $\overline{V_{1}^{\prime 2}}$ and $\overline{V_{2}^{\prime 2}}$ are proportional. This term is related to the density variations in combustion mixtures. In the figures of this section the notation used is $V x=V_{2}$, $V y=V_{1}, \operatorname{Re} y X X=\overline{V_{1}^{\prime 2}}, \operatorname{Re} y Y Y=\overline{V_{2}^{\prime 2}}$ and $\operatorname{Re} y X Y=\overline{V_{1}^{\prime} V_{2}^{\prime}}$.

The flowfield structure of two cases using Nitrogen in the center jet was approached in order to analyze the behavior of the turbulent properties. These cases, presented

Table 1. Parameters of flowfield for cases considered.

\begin{tabular}{ccccccc}
\hline Case Gas & $\begin{array}{c}\text { Jet flow } \\
\left(\mathrm{Nm}^{3} / \mathrm{h}\right)\end{array}$ & Jet vel. $(\mathrm{m} / \mathrm{s})$ & $\begin{array}{c}\text { Jet } \\
\text { Reynolds } \\
\text { number }\end{array}$ & $\begin{array}{c}\text { Air Vel. } \\
(\mathrm{m} / \mathrm{s})\end{array}$ & $\begin{array}{c}\mathrm{dt} \\
(\mu \mathrm{s})\end{array}$ \\
\hline 1 & $N_{2}$ & $1.90 \pm 0.15$ & $13.30 \pm 0.90$ & $6066 \pm 425$ & $4.00 \pm 0.01$ & 27 \\
2 & $N_{2}$ & $0.60 \pm 0.05$ & $4.20 \pm 0.25$ & $1915 \pm 135$ & $8.00 \pm 0.02$ & 45 \\
\hline
\end{tabular}

as 1 and 2 in Table 1, was choose to represent different situations on the recirculation zone. First case, has the fuel quantity of movement three times higher than the case 2 , whereas the flowfield is dominated by the jet. In other hand, in the second case occurs the air advantage, in relation of the case 1 .

\subsection{Analysis of the Cases}

The flowfield structure of two cases using Nitrogen in the center jet was approached in order to analyze the behavior of the turbulent properties. These cases, presented as 1 and 2 in Table 1, was choose to represent different situations on the recirculation zone. First case, has the fuel quantity of movement three times higher than the case 2 , whereas the flowfield is dominated by the jet. In other hand, in the second case occurs the air advantage, in relation of the case 1 .

\subsubsection{Instantaneous Flowfield Analysis}

Figure 1(a) shows the vector velocity field of the case 1 flowfield. Three instantaneous images of the velocity distribution are presented, where the center jet blows out the wake region. The quantity of movement of the jet is practically the double of the air flowfield leading these characteristics, which also confirm high symmetry of the flowfield.

Figure 1(b) shows the behavior of the velocity flowfield to the case 2. The instantaneous measures introduces the low fluctuation of the air flowfield region and in the jet downward of $y=20 \mathrm{~mm}$ from which the jet disappear. This behavior is due the quantity of movement in the jet be smaller than in the air flowfield, if compared to the case 1 .

\subsubsection{Mean Flowfield Analysis}

Figure 2 show the mean velocity flowfield distribution overlapped by the streamlines. In Figure 2(a), the center jet is predominant by the whole longitudinal direction of the measurement window, creating a toroidal vortex centered in $y=20 \mathrm{~mm}$, upward this there is a compression zone in the flowfield.

In the mean flowfield of the case 2, showed in the Figure 2(b), toroidal recirculation zones are found, which produce two stagnation points, along of the $x / D=0$. The streamlines draft these points, in the symmetry axis, between 30 and $60 \mathrm{~mm}$ upward of the burner surface. Thus, two recirculation zones can be seem, first, has a smaller diameter than the second and is placed in the vicinity of the jet, $y=10 \mathrm{~mm}$, in the boundary between jet and wake. Second, centered in $y=30 \mathrm{~mm}$ occupies the whole wake in the transversal direction. In this case the flowfield is dominated by the wake, differently of the case 1 , which has the center jet predominant. 

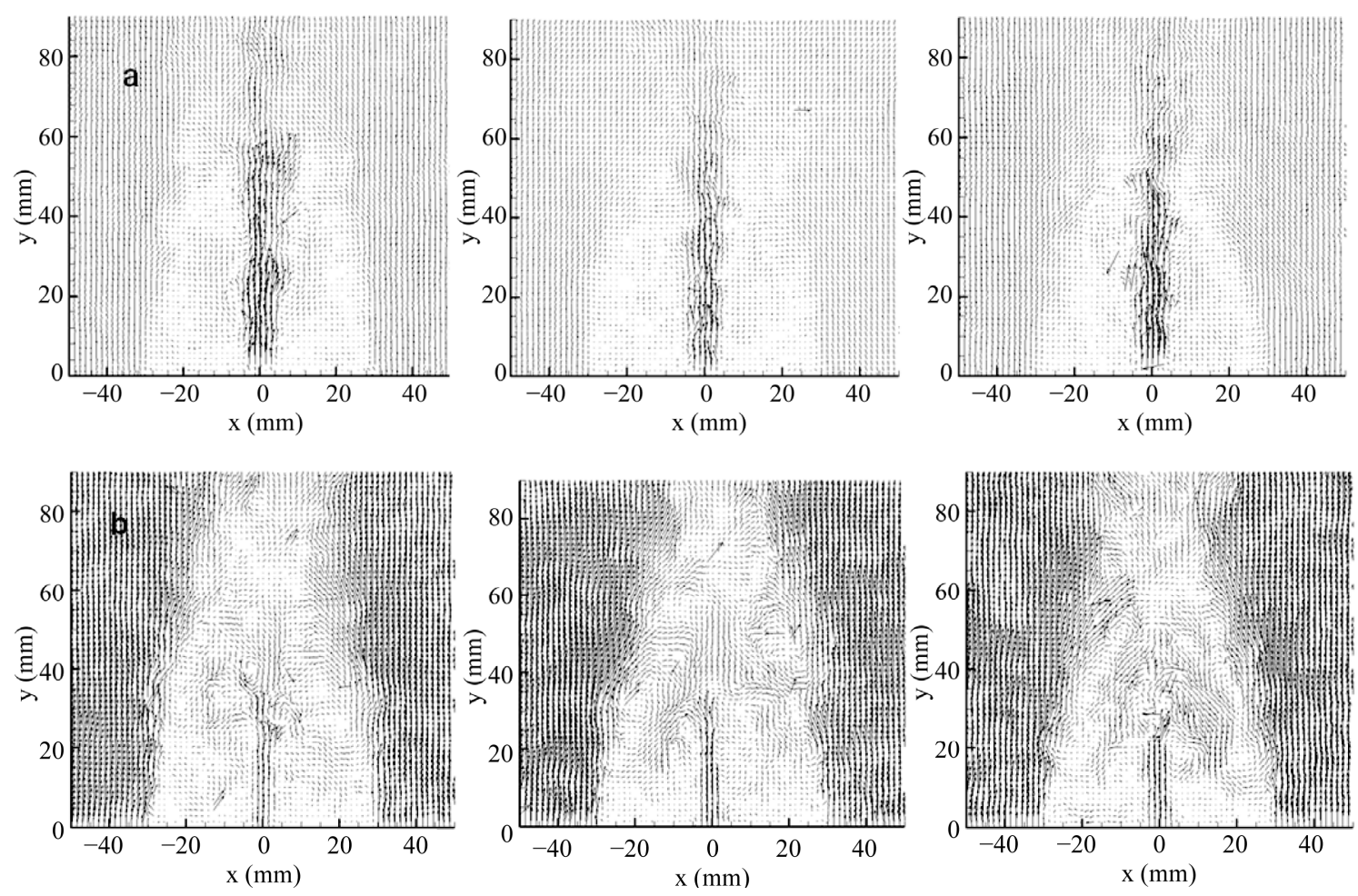

Figure 1. Case 1 (a); 2 (b): Instantaneous velocity.
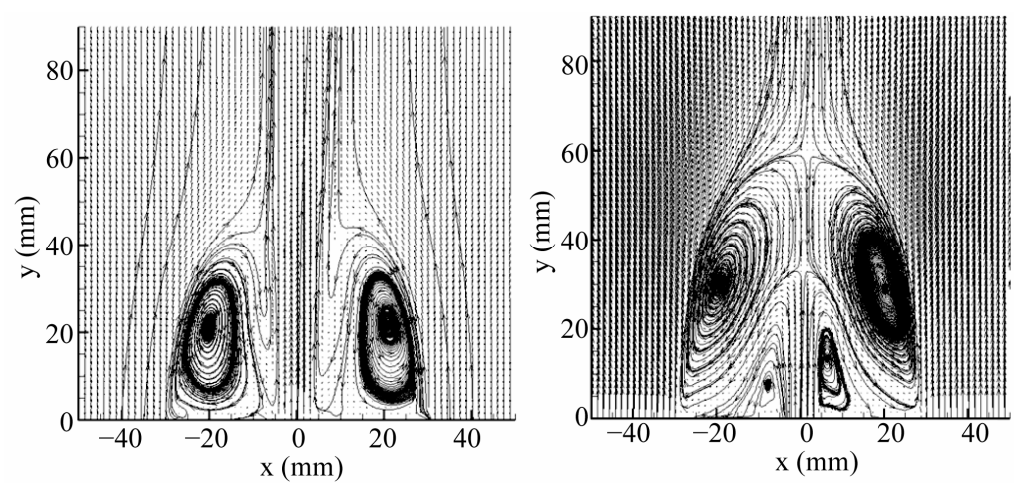

Figure 2. Case 1 (a); case 2 (b): Mean velocity vectors and streamlines.

\subsubsection{Analysis of Case 1}

The longitudinal velocity component, $V y$, showed in Figure 3(a), presents stead values close to $4 \mathrm{~m} / \mathrm{s}$ in the air flowfield region, $(|x / D|>0.50)$, reaching $15 \mathrm{~m} / \mathrm{s}$ in the center jet region $10 \mathrm{~mm}$ upward from the burner nozzle. This value decreases along to the center line until 5 $\mathrm{m} / \mathrm{s}$ in $y=70 \mathrm{~mm}$. In the wake region,

$0.10<|x / D|<0.50$, the $V y$ values decreases until comes to take negative values between $y=20$ and $40 \mathrm{~mm}$, due the recirculation zone and, increasing again in the jet surrounds. In this case, the wake works as a boundary between jet and air flowfields.

In the wake region, the transversal component of velocity, $V x$, showed in the Figure 3(b), reaches highest values in the recirculation region if compared with values in the center of the jet. To $|x / D|>0.50, V x$ shows the drag process of the air in the wake produced by the bluffbody. Figure 3(b) shows the abrupt variations of the $V x$ in the jet and wake boundary, with values slightly higher in the positive side, i.e., in $x / D=0.1$.

The experimental results show, as expected in this flowfield strongly dominated by the jet, that the values of Reynolds stress tensor components, $\operatorname{Re} y Y Y$ and $X X$, showed in the Figures 3(c) and (d), respectively, are direct related with the high flowfield mean strain rates and, also each other. The $\operatorname{Re} y X Y$ component, showed in Figure 3(e), is related with the flowfield shear rate.

The Reynolds stress tensor components measured confirm the characteristics of jet flowfield, in this first case, which shows the higher values along the boundary be- 

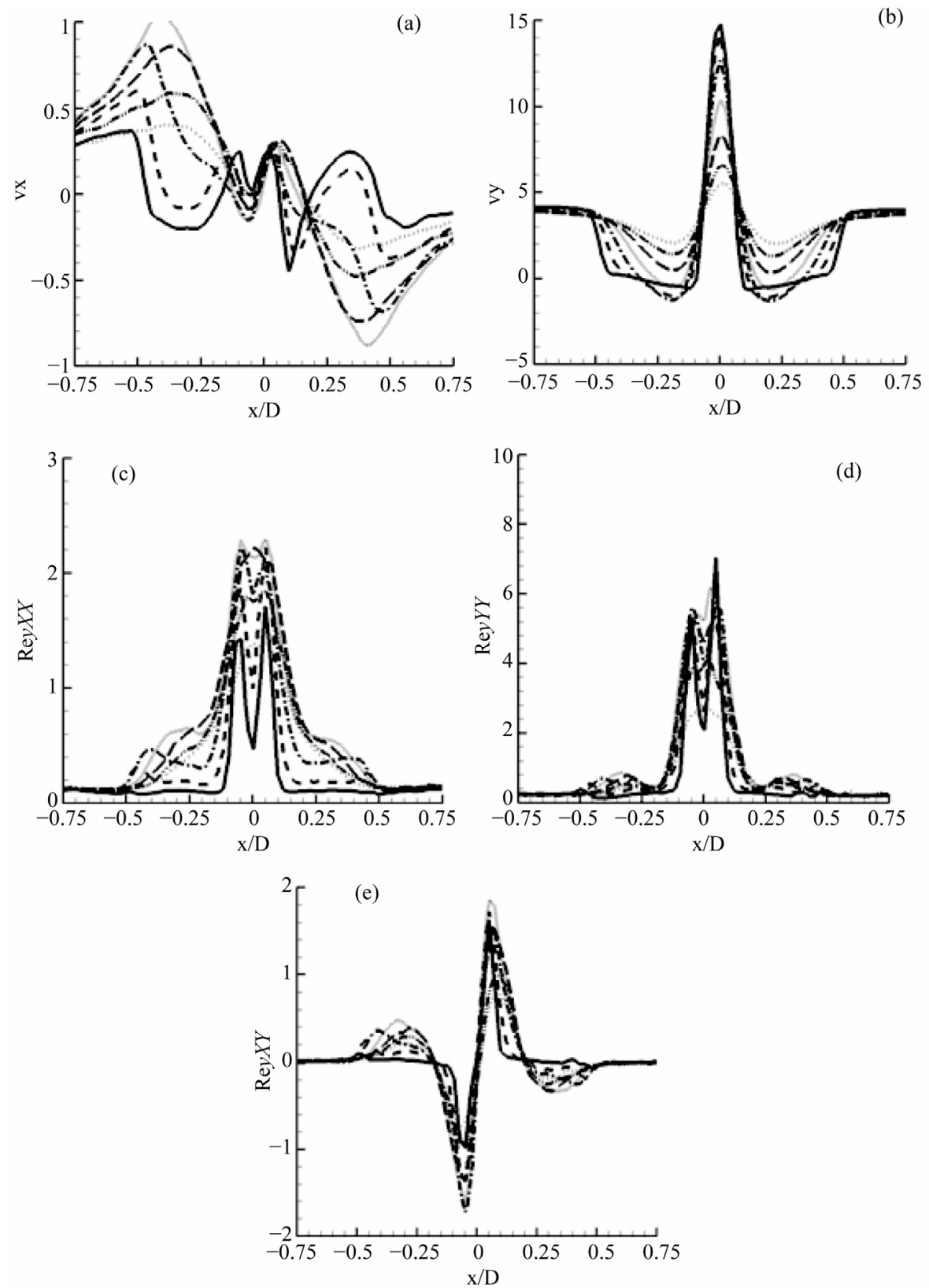

Figure 3. Case 1: Evolution in transversal direction $(x)$, to longitudinal positions spaced $10 \mathrm{~mm}$ from the burner surface, of

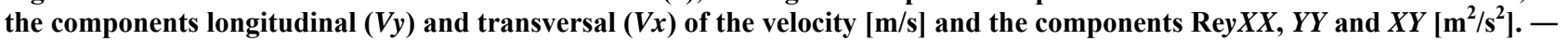
$10,--20, \cdots 30,-\bullet-40,--50,-\bullet \cdot 60, \bullet \cdots 70 \mathrm{~mm}$.

tween jet and wake, where the highest strain rates are found. The components, $\operatorname{Re} y X X, Y Y$, are small in the air flowfield region, circa $0.1 \mathrm{~m}^{2} / \mathrm{s}^{2}$, where the velocity gradient is zero, see Figure 3(a). In this same region $\operatorname{Re} y X Y$ is null, indicating that there is turbulence isotropy. The stress values increases to $0.5 \mathrm{~m}^{2} / \mathrm{s}^{2}$ in the wake region, mainly upward to the recirculation zone, $40<y<$ $70 \mathrm{~mm}$. The maximum values occurs in the jet/wake boundary, where $\operatorname{Re} y X X=2, \operatorname{Re} y Y Y=7$ and $\operatorname{Re} y X Y=2$ $\mathrm{m}^{2} / \mathrm{s}^{2}$. Note that, in the central side of the jet the values of Reynolds stress components decreases close the burner surface, i.e., to $y<20 \mathrm{~mm}$.

An analysis of the $\operatorname{Re} y X Y$ and $\operatorname{Re} y X X$ results showed in the Figures 3(c) and (d), allows verifying that the Boussinesq hypothesis is satisfied, due two reasons. First, both stress values corresponds to the higher mean strain rate, second, there is a direct relation between $\operatorname{Re} y X Y$ and Re $y X X$. The Figure 3(e) analysis also allows verifying that there is a proportionality between the mean strain rate for shear and $\operatorname{Re} y X Y$. Thus, within the experimental uncertainty of the results, the Boussinesq hypothesis to incompressible flowfields is satisfied in the whole 
region. In the wake, $0.10<|x / D|<0.50$, seems occurs a low deviation to the normal stress proportionality, nevertheless, this is due the difficulty in measure accurately the small values of $V x$ if compared with $V y$ values.

\subsubsection{Analysis of Case 2}

The values of $V y$ keep steady close to $8 \mathrm{~m} / \mathrm{s}$ in the air flowfield region, $|x / D|>0.50$. Along the center line this velocity component reaches $7 \mathrm{~m} / \mathrm{s}$ in the maximum at 10 $\mathrm{mm}$ upward to the burner surface, decreasing drastically to $-2 \mathrm{~m} / \mathrm{s}$ within $40<y<60 \mathrm{~mm}$. Upward to $y=70 \mathrm{~mm}$ the mixture region becomes homogeneous and tends to be dominated by the air flowfield, which has $V y=1 \mathrm{~m} / \mathrm{s}$.

Figure 4(a) shows the evolution of the values from the measurements results performed in the case 2 to several heights above the burner surface. The velocity component, $V y$, allows to note that upward to $y=30 \mathrm{~mm}$ there is a strong interaction between jet and air along the whole wake region, producing intense turbulence, evident in the Reynolds stress profiles. The jet is broken in $y$
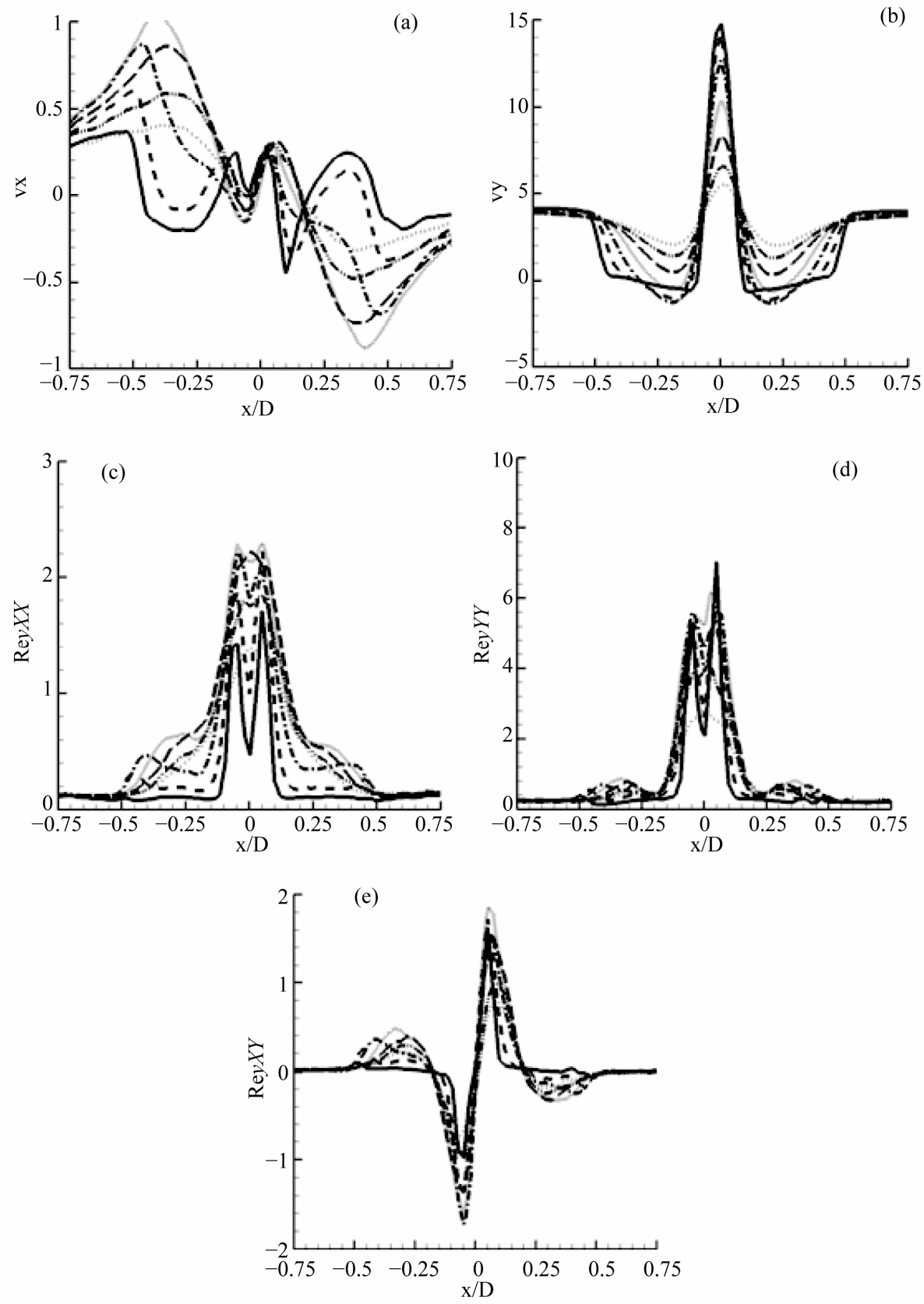

Figure 4. Case 2: Evolution in transversal direction $(x)$, to longitudinal positions spaced $10 \mathrm{~mm}$ from the burner surface, of the components longitudinal $(V y)$ and transversal $(V x)$ of the velocity $[\mathrm{m} / \mathrm{s}]$ and the components $\operatorname{Rey} X X, Y Y$ and $X Y\left[\mathrm{~m}^{2} / \mathrm{s}^{2}\right]$. $10,---20, \cdots \cdot 30,-\bullet-40,---50,-\bullet 60, \cdots 70 \mathrm{~mm}$. 
$=40 \mathrm{~mm}$, where a jet and air mixture is intense, consistent with the jet scattering process occurred in the wake region. In the wake region, the transversal component of velocity, $V x$, showed in the Figure 4(b), reaches highest values in the recirculation region near the wake and air boundary. Figure 4(b) shows the minor variations of the $V x$ in the jet and wake boundary.

The Reynolds stress tensor components, Figures 4(c)(e), confirm that, in the case 2, the flowfield characteristics are dominated by the wake. The component $\operatorname{Re} y X Y$ shows the local maximum values, $2 \mathrm{~m}^{2} / \mathrm{s}^{2}$, in $|x / D|$ $=0.50$ and $|x / D|=0.10$. These values increase gradually until $y=40 \mathrm{~mm}$, in particular, reaches values close to those achieved in case 1 , whose jet is predominant, circa $2 \mathrm{~m}^{2} / \mathrm{s}^{2}$. The comparison of Figures 4(c) and (d) suggests that ReyXX and ReyYY are not proportional, i.e., the Boussinesq hypothesis should not be used as model in order to simulate this flowfield.

The component ReyXX is practically uniform in the wake region, where the highest measured values evolutes from downward to upward, which intensify and spread in the region that air and wake mixture occurs.

The component ReyXY presents a maximum absolute value of in $1 \mathrm{~m}^{2} / \mathrm{s}^{2}$ wake region. From downward to upward Re $y X Y$ is transported from the air and wake boundary, $|x / D|=0.50$, in direction to the center, $|x / D|=0$. This is other indicative that the flowfield structure is dominated by the wake.

The Boussinesq hypothesis is not sufficient to description of the turbulent transport of the case 2 flowfield, instead of the case 1 . However, a mathematic model designed to numerical simulation of this flowfield should use a Reynolds stress transport model.

\section{Conclusions}

The detailed characterization of the turbulent flowfield requires, necessarily, the velocity distribution with a spatial resolution such as allows to analyze the interaction of the vortex in the large scale and the turbulence isotropy. The detailed velocity field distribution allows the development of the new models, which are able to describe the different combustion regimes.

Future works intend to employ the stereo PIV technique, which allows a reconstruction of the measurements toward obtain $3 \mathrm{D}$ results, in order to produce more information, i.e., the nine components of the Reynolds tensor. Thus, will be possible the calculation of those three anisotropy components [17].

In this work were presented results of the turbulent flowfield characterization from a nitrogen jet and annular air in coflow configuration, stabilized upward a bluffbody burner. These results are important regarding the representation of the industrial conditions and prepare to analyze reaction conditions. The main advantages of the optical technique used in this work, PIV, are the nonintrusive measurements, the high resolution images results and the low uncertainty. The achieved results allow to visualization of the flowfield detailed structure, designed for the vector velocity field. Furthermore, the thorough measurements uncertainty analysis yielded $6 \%$.

The instantaneous images allow to note that as the flowfield turbulence quantity increases the variations of the intern structure grows up. These local characteristics hamper the numerical simulations of the flowfield. The data results about the velocity distribution provide information to development and validation of the new computational models in order to improve combustion systems projects and numerical simulations.

\section{REFERENCES}

[1] M. Raffel, C. E. Willert, S. T. Wereley and J. Kompenhans, "Particle Image Velocimetry, a Praticle Guide," Göttingen, Springer, 2007, p. 448.

[2] J. Westerweel, "Fundamentals of Digital Particle Image Velocimetry," Measurement Science and Technology, Vol. 8, No. 12, 1997, pp. 1379-1392. doi:10.1088/0957-0233/8/12/002

[3] N. R. Caetano, "Estudo Experimental de Chamas Turbulentas não Pré-Misturadas Empregando Simultaneamente as Técnicas de Diagnóstico Laser PLIF e PIV," Ph.D. Thesis, Mechanical Engineering Department, PUCRio, Rio de Janeiro, 2012, p. 180.

[4] B. Böhm, C. Heeger, I. Boxx, W. Meier and A. Dreizler, "Time-Resolved Conditional Flow Field Statistics in Extinguishing Turbulent Opposed Jet Flames Using Simultaneous High Speed PIV/OH-PLIF," Proceedings of the Combustion Institute, Vol. 32, No. 2, 2009, pp. 16471654. doi:10.1016/j.proci.2008.06.136

[5] P. S. Kothnur, M. S. Tsurikov, N. T. Clemens, J. M. Donbar and C. D. Carter, "Planar Imaging of $\mathrm{CH}, \mathrm{OH}$, and Velocity in Turbulent Non-Premixed Jet Flames," Proceedings of the Combustion Institute, Vol. 29, No. 2, 2002, pp. 1921-1927. doi:10.1016/S1540-7489(02)80233-4

[6] A. M. Steinberg, J. F. Driscoll and S. L. Ceccio, "Temporal Evolution of Flame Stretch Due to Turbulence and the Hydrodynamic Instability," Proceedings of the Combustion Institute, Vol. 32, No. 2, 2009, pp. 1713-1721. doi:10.1016/j.proci.2008.05.003

[7] M. Shimura, T. Ueda, G. Choi, M. Tanahashi and T. Miyauchi, "Simultaneous Dual-Plane CH PLIF, SinglePlane OH PLIF and Dual-Plane Stereoscopic PIV Measurements in Methane-Air Turbulent Premixed Flames," Proceedings of the Combustion Institute, Vol. 33, No. 1, 2011, pp. 775-782. doi:10.1016/j.proci.2010.05.026

[8] G. Troiani, M. Marrocco, S. Giammartini and C. M. Casciola, "Counter-Gradient Transport in the Combustion of a Premixed CH4/Air Annular Jet by Combined PIV/ OH-LIF," Combustion and Flame, Vol. 156, No. 3, 2009, pp. 608-620. doi:10.1016/i.combustflame.2008.12.010 
[9] L. E. A. Huapaya, "Caracterização Numérica e Experimental de Uma Chama Turbulenta não Pré-Misturada," Ph.D. Thesis, Mechanical Engineering Department, PUCRio, Rio de Janeiro, 2008, p. 169.

[10] P. T. Lacava and C. A. Martins, "Métodos Experimentais de Análise Aplicados à Combustão," Papel Brasil, 2010.

[11] A. P. S. Freire, A. Ilha and R. Breidenthal, "Turbulência," 5th Turbulence and Transition School, IME, Rio de Janeiro, 2006.

[12] P. Petersson, J. Olofsson, C. Brackman, H. Seyfried, J. Zetterberg, M. Richter, M. Aldén, M. A. Linne, R. K. Cheng, A. Nauert, D. Geyer and A. Dreizler, "Simultaneous PIV/OH-PLIF, Rayleigh Thermometry/OH-PLIF and Stereo PIV Measurements in a Low-Swirl Flame," Applied Optics, Vol. 46, No. 9, 2007, pp. 3928-3936. doi:10.1364/AO.46.003928
[13] E. T. Whittaker, "Expansions of the Interpolation-Theory," Proceedings of the Royal Society, Vol. 35, 1915, p. 181.

[14] T. Roesgen, "Optimal Subpixel Interpolation in Particle Image Velocimetry," Experiments in Fluids, Vol. 35, No. 3, 2003, pp. 252-256. doi:10.1007/s00348-003-0627-8

[15] S. J. Kline and F. A. McClintock, "Describing Uncertainties in Single-Sample Experiments," Mechanical Engineering, 1953.

[16] R. J. Moffat, "Contributions to the Theory of Single-Sample Uncertainty Analysis," Journal of Fluids Engineering, Vol. 104, No. 2, 1982, pp. 250-258. doi:10.1115/1.3241818

[17] S. B. Pope, "Turbulent Flows," Cambridge, 2000, p. 749. doi:10.1017/CBO9780511840531 